\title{
Distribución y Conservación de Magnolia (Magnoliaceae) en Cuba
}

\section{Distribution and conservation of Magnolia (Magnoliaceae) in Cuba}

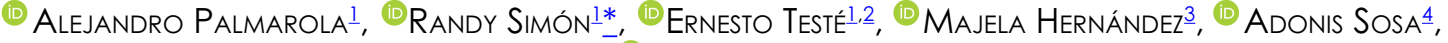 \\ (1) Yenia Molina ${ }^{4}$, ${ }^{\circ}$ Luis Roberto González-Torres 5
}

\author{
I Jardín Botánico Nacional, Universidad de La Habana, La Habana, Cuba. \\ ${ }^{2}$ Ecologie Systématique et Evolution, Université Paris-Saclay, Orsay, France. \\ ${ }^{3}$ Departamento de Biología Vegetal, Facultad de Biología, Universidad de La Habana, La Habana, Cuba. \\ ${ }^{4}$ Instituto de Investigaciones Agro-Forestales. Estación Experimental Agro-Forestal Guisa, Guisa, Granma, Cuba. \\ ${ }^{5}$ Departament of Biology, Douglas College, New Westminister, Canada. \\ *Autor por correspondencia: rsimonvallejo@gmail.com
}

\section{Resumen}

Antecedentes: Gran parte de las evaluaciones del estado de conservación de los taxones de Magnoliaceae en Cuba se han basado en inferencias sobre su distribución y tamaños poblacionales. No obstante, estas investigaciones se han enfocado en un taxón o subsección.

Preguntas: ¿Cómo se distribuyen los taxones nativos de Magnoliaceae en Cuba? ¿Cuál es su estado de conservación actual?

Especies de estudio: Taxones nativos de Magnoliaceae.

Sitio y años de estudio: Cuba, 1884-2020.

Métodos: Se utilizaron 3,481 puntos de presencia. A partir de las coordenadas de cada taxón se mapeó la distribución real, se registró la formación vegetal, altitud, área protegida donde se localizaban, se calculó la extensión de presencia, el área de ocupación y la densidad. Se resumió el estado de conservación de los taxones a partir de sus categorizaciones actuales.

Resultados: Magnoliaceae en Cuba se distribuye a altitudes promedios elevadas en los macizos montañosos Guamuhaya, Sierra Maestra y Nipe-Sagua-Baracoa, asociada fundamentalmente a los bosques siempreverdes; con excepción de Magnolia virginiana subsp. oviedoae que se encuentra al nivel del mar y en formaciones vegetales de ciénagas. Se encontró una gran representatividad en áreas protegidas. Todas las magnolias cubanas se encuentran amenazadas, fundamentalmente debido a la tala, la modificación del hábitat por la presencia de especies exóticas invasoras y la existencia de plantaciones forestales.

Conclusiones: En sentido general, los patrones de distribución responden a lo descrito para el género en Cuba y el Caribe. Exceptuando Magnolia minor, las categorías de amenazas de las magnolias cubanas cambiaron con respecto a las categorizaciones anteriores.

Palabras claves: distribución real, especies amenazadas, magnolias.

Abstract

Background: Most of the assessments of the conservation status of Magnoliaceae taxa in Cuba have been based on inferences about their distribution and population sizes. However, these investigations have focused on one taxon or subsection.

Questions: How are native Magnoliaceae taxa distributed in Cuba? What is the current state of conservation?

Study species: Six native species of Magnoliaceae

Site and years of study: Cuba, 1884-2020.

Methods: 3,481 points of presence were used. From the coordinates of each taxon, the real distribution was mapped, the plant formation, altitude and protected area where they were located was recorded, the extension of presence, the area of occupation and the density were calculated. The conservation status of the taxa was summarized from their current categorizations.

Results: Magnoliaceae in Cuba is distributed at high average altitudes in the Guamuhaya, Sierra Maestra and Nipe-Sagua-Baracoa mountain ranges, mainly associated with evergreen forests; with the exception of Magnolia virginiana subsp. oviedoae found at sea level and in swamp vegetation. A great representativeness was found in protected areas. All Cuban magnolias are threatened, mainly due to logging, habitat modification due to the presence of invasive alien species and the existence of forest plantations.

Conclusions: In general, the distribution patterns respond to what has been described for the genus in Cuba and the Caribbean. With the exception of Magnolia minor, the threat categories for Cuban magnolias changed with respect to the previous categorizations.

Keywords: actual distribution, threatened species, magnolias.

Este artículo se encuentra bajo los términos de la licencia Creative Commons Attribution License CCBY-NC (4.0) internacional. https://creativecommons.org/licenses/by-nc/4.0/ 


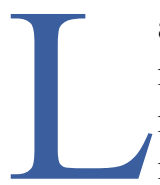

a familia Magnoliaceae Juss. comprende cerca de 350 especies de árboles y arbustos distribuidas en las regiones templadas, subtropicales y tropicales del Este y Sureste de Asia y en el continente americano (Cires et al. 2013, Vázquez-García et al. 2016a). Según las últimas evaluaciones alrededor de 200 especies de magnolias se encuentran en alguna categoría de amenaza (Rivers et al. 2016, IUCN 2019). Tal situación ha sido causada fundamentalmente por la pérdida de hábitats debido fundamentalmente a la expansión agrícola y la ganadería, el uso no sostenible de su madera, la baja regeneración natural y la distribución restringida que presentan muchas de las magnolias (Gutiérrez-Lozano et al. 2020, Muñiz-Castro et al. 2020).

Los estudios demográficos en hábitats naturales muestran como son afectadas las poblaciones de una especie y su resiliencia (Vásquez-Morales 2015, Sánchez-Velásquez et al. 2016, Wiehle et al. 2016). Estos se complementan con la obtención detallada y precisa de información sobre su distribución, lo que se ha convertido en un paso crítico para la conservación (Paredes-García et al. 2011). Conocer la distribución de una especie permite desarrollar estrategias efectivas de conservación in situ y ex situ (Menon et al. 2010, Paredes-García et al. 2011). Estos estudios se enfocan en localizar zonas de búsqueda, de reforzamiento y de conservación más estricta.

En Cuba, según la clasificación más actualizada para el género Magnolia L., existen 7 taxones endémicos (Greuter \& Rankin 2017), distribuidos en tres secciones: Magnolia, Splendentes Dandy ex A. Vázquez, y Talauma (Juss.) Baill. (Wang et al. 2020). Magnolia virginiana subsp. oviedoae A. Palmarola, M. S. Romanov \& A. V. Bobrov. es el único taxón cubano de la sección Magnolia. La sección Talauma cuenta con tres taxones: M. minor (Urb.) Govaerts, M. oblongifolia (León) Palmarola y M. orbiculata (Britton \& P. Wilson) Palmarola; y la sección Splendentes también con tres representantes: M. cubensis subsp. acunae Imkhan., M. cubensis Urb. subsp. cubensis y M. cristalensis Bisse (Palmarola et al. 2016, Greuter \& Rankin 2017).

Las Magnolias del Neotrópico, incluidas las del Caribe y las de México y Centro América presentan un patrón marcado de distribución alopátrica (Vázquez-García 1994, Vázquez-García et al. 2015, 2016a, 2018), con algunas excepciones (Vázquez-García et al. 2018, Veltjen 2020). Las magnolias cubanas, al igual que otras magnolias a nivel mundial y varios taxones de la flora de Cuba, presentan un patrón de distribución agregado y bajos números de individuos, característicos de especies con requerimientos ambientales estrictos (Li et al. 2017, Palmarola et al. 2018). Estas características, sumadas a la deforestación, la fragmentación de sus hábitats, el desarrollo agroforestal y la tala furtiva, han traído consigo que todos los taxones cubanos de Magnolia presenten alguna categoría de amenaza según los criterios de la IUCN (González-Torres et al. 2016, Rivers et al. 2016). En las hojas de taxón de las magnolias cubanas, publicadas por González-Oliva et al. (2015), los autores basan gran parte de las evaluaciones del estado de conservación de estos taxones en inferencias sobre su distribución. Luego de esta evaluación, varias investigaciones se han enfocado en profundizar en la ecología poblacional, taxonomía y aspectos sobre la distribución de la familia en Cuba (Palmarola et al. 2016, 2017, 2018, Testé 2018, Testé et al. 2019). De ahí que las últimas evaluaciones (Hernández et al. en prensa a, b, c, d, e, f,) de sus categorías de amenaza reflejan la importancia de basar las categorizaciones en datos más precisos. Dada la ausencia hasta la fecha de una investigación que resuma y actualice los resultados obtenidos en los últimos cinco años, sobre la distribución y ecología de las magnolias cubanas y, dada la importancia de estos datos para evaluaciones futuras, en el presente estudio se proponen como objetivos: actualizar y caracterizar la distribución geográfica de los taxones de Magnolia endémicos de Cuba y resumir su estado de conservación.

\section{Materiales y métodos}

Distribución geográfica. La distribución se describió mediante el empleo de datos de campo y de herbario (Tabla 1). Los primeros fueron obtenidos en expediciones realizadas entre 2010 y 2019, en las cuales se censaron y georreferenciaron (GPS Garmin $\pm 2 \mathrm{~m}$ ) los individuos. Los puntos de presencia de Magnolia cubensis subsp. acunae y Magnolia virginiana subsp. oviedoae fueron obtenidos de Palmarola et al. (2018) y Testé (2018), respectivamente.

Los datos de herbario fueron tomados de especímenes depositados en B, BSC, BM, FTG, G, HAC, HAJB, JE, K, LE, NY, P, S y US (acrónimos de herbarios según Thiers 2020) con el fin de completar la información de aquellas 
localidades no visitadas. Se estimó la localización más precisa posible para los materiales que no poseían coordenadas geográficas. Todos los registros de presencia extraídos de los herbarios fueron georreferenciados en coordenadas decimales utilizando Google Maps (google.es/maps). Para describir la distribución real, se confeccionaron mapas a partir de las coordenadas de cada taxón, usando como base el modelo digital de elevación (MDE) obtenido de WorldClim (worldclim.org), y el mapa de áreas protegidas de Cuba (CNAP 2014).

Tabla 1. Registros de presencia utilizados para la descripción de la distribución de los taxones cubanos de Magnolia, obtenidos en expediciones realizadas entre 2010 y 2019 y datos de herbario.

\begin{tabular}{lccc}
\hline Taxón & Expediciones & Herbario & Total \\
\hline Magnolia virginiana subsp. oviedoae & 1,350 & 0 & 1,350 \\
Magnolia cubensis subsp. acunae & 447 & 0 & 447 \\
Magnolia cubensis subsp. cubensis & 663 & 0 & 663 \\
Magnolia cristalensis & 186 & 11 & 197 \\
Magnolia minor & 367 & 4 & 371 \\
Magnolia oblongifolia & 396 & 4 & 400 \\
Magnolia orbiculata & 53 & 0 & 53 \\
\hline
\end{tabular}

La extensión de presencia (EP) se calculó como el polígono mínimo convexo que contuvo todos los registros conocidos de cada taxón (IUCN 2019). El área de ocupación (AO) se calculó como la sumatoria del área de las cuadrículas ocupadas por cada uno de los taxones. El área de la cuadrícula se ajustó a $1 \mathrm{~km}^{2}$ como recomienda el Grupo de Especialistas de Plantas Cubanas, y que representa la cuarta parte de la recomendada por la IUCN (2019). Esta área constituye la medida adecuada en plantas cubanas para indicar el área de hábitat apropiado asociado a un registro de presencia de los taxones evaluados. Ambos parámetros (EP y AO) fueron calculados en la plataforma GeoCAT (Geospatial Conservation Assessment Tool) (Bachman et al. 2011; geocat.kew.org). Se calculó la densidad para cada taxón a partir de los puntos de presencia y la EP. Los valores de formación vegetal y área protegida se extrajeron, usando ArcGis 10.2 (Esri 2013), en cada punto de presencia a partir del mapa de vegetación de Estrada et al. (2019) y el mapa de áreas protegidas del CNAP (2014), respectivamente. Estrada et al. (2019), basa su clasificación en las categorías originales definidas por Capote \& Berazaín (1984) y le agrega 17 nuevas clases tomadas de otros autores cuyas clasificaciones contenían elementos que podían ser delimitados mediante análisis de Sistemas de Información Geográfica (SIG). Para cada taxón, a partir de los puntos de presencia, se calculó el porcentaje de inclusión en áreas protegidas.

A partir del modelo digital de elevación se extrajeron los valores promedios, mínimos y máximos de altitud para cada taxón, usando ArcGis 10.2 (Esri 2013). Se comprobó la normalidad de los datos de esta variable mediante la prueba de Shapiro-Wills y la homogeneidad de varianza a través de la prueba de Levene. Debido al incumplimiento de ambas premisas se utilizó una prueba de Kruskal-Wallis y la prueba de Dunn como análisis de comparación múltiple de media a posteriori, para comparar las diferencias de los promedios de altitud entre los taxones. Estos procedimientos fueron realizados en el entorno R (R Core Team 2020) mediante la plataforma web univariados gallery github.com/VMRA/univariados_gallery), con un nivel de significación estadística de $p=0.01$.

Conservación. La categoría de amenaza de cada uno de los taxones de Magnolia nativos de Cuba se extrajo de sus hojas de taxón más actualizadas (Hernández et al. en prensa a, b, c, d, e, f, g). Se resumieron, además, los criterios utilizados para asignarlos y las principales amenazas de los taxones en el hábitat donde se desarrollan. Se muestran los criterios de amenazas utilizados por los autores (IUCN 2019) para la re-categorización: Distribución geográfica representada como extensión de presencia (B1) y/o área de ocupación (B2); pequeño tamaño poblacional y disminución $(\mathrm{C})$; población muy pequeña o restringida (D). 


\section{Resultados}

Los taxones cubanos de Magnolia habitan en la Ciénaga de Majaguillar y en tres de los principales macizos del país: Guamuhaya, Sierra Maestra y Nipe-Sagua-Baracoa (Figura 1). Magnolia virginiana subsp. oviedoae se encuentra en la zona occidental del país en la Ciénaga de Majaguillar, Matanzas (Figura 1A). Magnolia cubensis subsp. acunae se localiza más al este, en Guamuhaya y es la única magnolia de montaña cuya distribución no se superpone con la de otra especie del grupo (Figura 1B). En la Sierra Maestra conviven M. cubensis subsp. cubensis y M. orbiculata (Figura 1C), las cuales poseen similar distribución hacia la región oeste de la Sierra Maestra; mientras que, en la Sierra de la Gran Piedra solo se encuentra $M$. cubensis subsp. cubensis. Al norte de la región oriental cohabitan tres especies: M. minor, M. oblongifolia y M. cristalensis, de las cuales M. minor y M. oblongifolia comparten la mayoría de sus hábitats (Figura 1D).
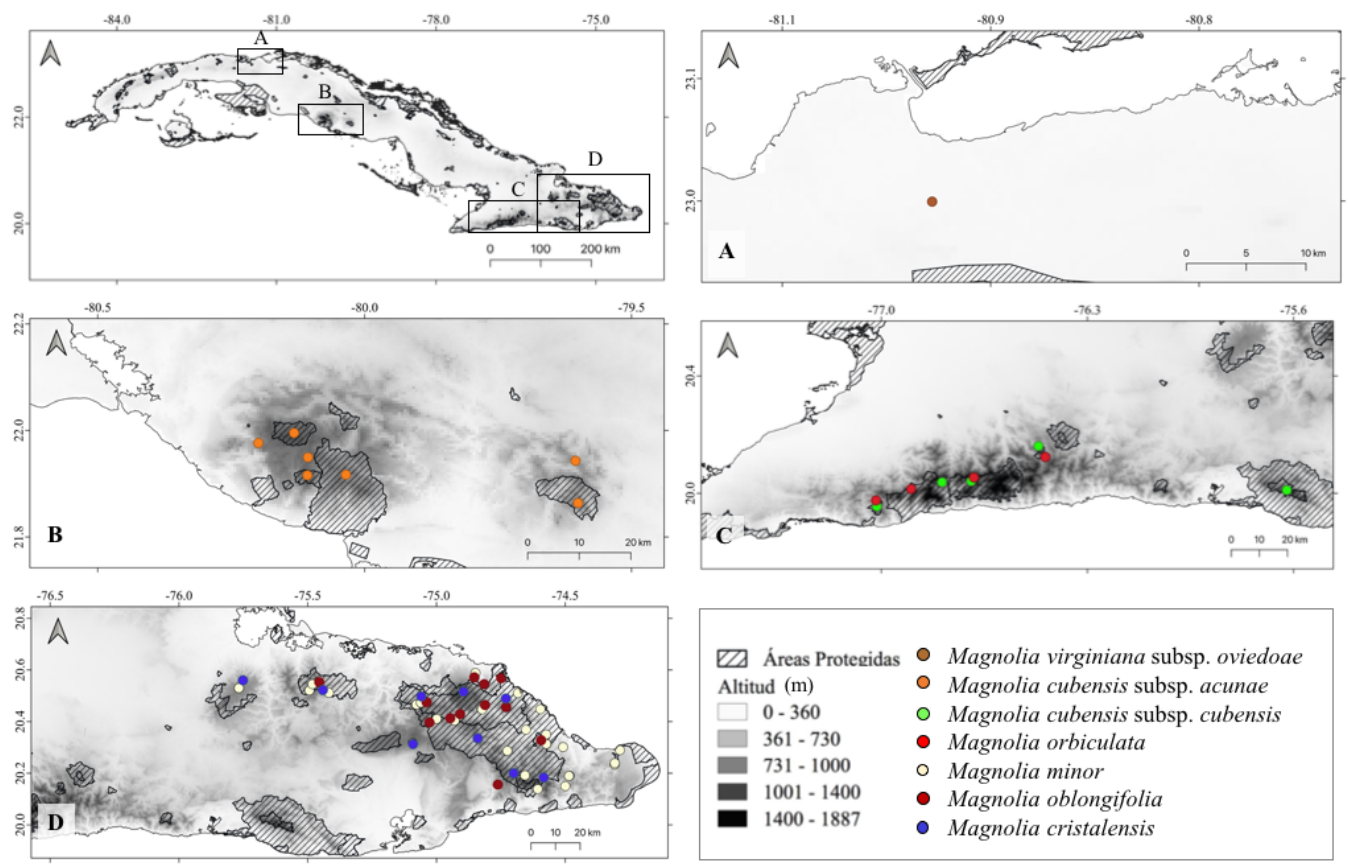

Figura 1. Distribución geográfica de los taxones cubanos de Magnolia. Ciénaga de Majaguillar (A), Macizo Guamuhaya (B), Macizo Sierra Maestra (C). Macizo Nipe-Sagua-Baracoa (D). Los puntos representan las localidades de presencia para cada uno de los taxones.

El patrón de distribución de Magnolia en Cuba resultó mayormente alopátrico dentro de una misma sección, el cual aplicó para cinco de los siete taxones de Magnolia en Cuba, Magnolia virginiana subsp. oviedoae (sect. Magnolia); M. cubensis subsp. acunae, M. cubensis subsp. cubensis y Magnolia cristalensis (sect. Splendentes) y $M$. orbiculata (sect. Talauma). Solamente se presentó un caso de simpatría entre dos especies de la sección Talauma: $M$. minor y M. oblongifolia, que comparten su hábitat en el $44.4 \%$ de las presencias.

La EP y el AO mostraron valores mínimos en Magnolia virginiana subsp. oviedoae con 3 y $6 \mathrm{~km}^{2}$, respectivamente. Mientras que M. minor presentó los mayores valores de EP $\left(3,778 \mathrm{~km}^{2}\right)$ y AO $\left(43 \mathrm{~km}^{2}\right)$. El mayor número de individuos por $\mathrm{km}^{2}$ lo presenta Magnolia virginiana subsp. oviedoae (450), mientras que los menores valores de densidad corresponden a Magnolia cubensis subsp. cubensis. Los taxones cubanos de Magnolia se registraron en 14 formaciones vegetales diferentes. De estas, la formación en la que se encuentra el mayor número de taxones es el bosque siempreverde mesófilo submontano, siendo el hábitat de cinco taxones. Magnolia minor se registró en ocho formaciones vegetales, siendo el taxón que se encuentra en mayor número de hábitats; mientras que los taxones 
que habitan en un menor número de formaciones vegetales (2), fueron M. orbiculata y Magnolia virginiana subsp. oviedoae (Tabla 2).

Tabla 2. Valores de Extensión de Presencia (EP), Área de Ocupación (AO), Densidad Poblacional (Dens.), Formación Vegetal (FV) y Áreas Protegidas (AP) de los taxones cubanos de Magnolia. Bosque pluvial montano (BPM), Bosques secundarios o plantaciones (BS), Bosque siempreverde mesófilo submontano (BSMS), Complejo de vegetación de mogote (CVM), Bosque nublado (BN), Matorral xeromorfo subespinoso sobre serpentinita (MXSS), Pluvisilva esclerófila submontana sobre serpentinita (PESS), Pluvisilva submontana sobre componente metamórfico (PSCM), Pluvisilva de baja altitud (PBA), Matorrales secundarios y seminaturales (MS), Herbazal de ciénaga (HC), Bosque de ciénaga (BC). Reserva Natural (RN), Parque Nacional (PN), Reserva Ecológica (RE), Elemento Natural Destacado (END), Reserva Florística Manejada (RFM), Paisaje Natural Protegido (PNP), Área Protegida de Recursos Manejados (APRM).

\begin{tabular}{|c|c|c|c|c|c|}
\hline Taxón & $\mathbf{E P}\left(\mathbf{k m}^{2}\right)$ & $\begin{array}{c}\text { AO } \\
\left(\mathbf{k m}^{2}\right)\end{array}$ & $\begin{array}{c}\text { Dens. } \\
\text { (ind./km) }\end{array}$ & FV & $\mathbf{A P}$ \\
\hline Magnolia virginiana subsp. oviedoae & 3 & 6 & 450 & $\begin{array}{l}\mathrm{BC} \\
\mathrm{HC}\end{array}$ & - \\
\hline Magnolia cubensis subsp. acunae & 442 & 28 & 1.01 & $\begin{array}{c}\text { BPM } \\
\text { BS } \\
\text { BSMS } \\
\text { CVM }\end{array}$ & $\begin{array}{l}\text { RE Lomas Banao } \\
\text { RE Pico San Juan } \\
\text { PNP Aguacate-Boca Carreras } \\
\text { PNP Topes de Collantes } \\
\text { PNP Hanabanilla }\end{array}$ \\
\hline Magnolia cubensis subsp. cubensis & 1,364 & 31 & 0.49 & $\begin{array}{c}\text { BN } \\
\text { BPM } \\
\text { BS } \\
\text { BSMS }\end{array}$ & $\begin{array}{l}\text { PN Pico la Bayamesa } \\
\text { PN Turquino } \\
\text { RE El Gigante } \\
\text { RE Pico Caracas } \\
\text { PNP Gran Piedra } \\
\text { APRM Reserva de la Biosfera Baconao } \\
\text { RE Loma del Gato }\end{array}$ \\
\hline Magnolia cristalensis & 2,154 & 29 & 0.09 & $\begin{array}{l}\text { BS } \\
\text { MXSS } \\
\text { PESS } \\
\text { PSCM }\end{array}$ & $\begin{array}{l}\text { PN Alejandro de Humboldt } \\
\text { PN Pico Cristal } \\
\text { RFM Pico Galán } \\
\text { APRM Reserva de la Biosfera Cuchillas del Toa. }\end{array}$ \\
\hline Magnolia minor & 3,778 & 43 & 0.1 & $\begin{array}{c}\text { BS } \\
\text { BSMS } \\
\text { CVM } \\
\text { MS } \\
\text { MXSS } \\
\text { PBA } \\
\text { PESS } \\
\text { PSCM }\end{array}$ & $\begin{array}{l}\text { RN Pinares de Montecristo } \\
\text { PN Alejandro de Humboldt } \\
\text { PN Pico Cristal } \\
\text { END Cañón del Yumurí } \\
\text { END Yunque de Baracoa } \\
\text { APRM Reserva de la Biosfera Cuchillas del Toa }\end{array}$ \\
\hline
\end{tabular}




\begin{tabular}{|c|c|c|c|c|c|}
\hline Taxón & $\mathbf{E P}\left(\mathbf{k m}^{2}\right)$ & $\begin{array}{c}\mathrm{AO} \\
\left(\mathbf{k m}^{2}\right)\end{array}$ & $\begin{array}{c}\text { Dens. } \\
\text { (ind./km) }\end{array}$ & $\mathbf{F V}$ & AP \\
\hline \multirow[t]{6}{*}{ Magnolia oblongifolia } & 2,353 & 33 & 0.17 & BS & PN Alejandro de Humboldt \\
\hline & & & & BSMS & PN Pico Cristal \\
\hline & & & & CVM & END Yunque de Baracoa \\
\hline & & & & MXSS & APRM Reserva de la Biosfera Cuchillas del Toa \\
\hline & & & & PBA & \\
\hline & & & & PESS & \\
\hline \multirow[t]{4}{*}{ Magnolia orbiculata } & 60 & 10 & 0.88 & BPM & PN Turquino \\
\hline & & & & BSMS & PN Pico la Bayamesa \\
\hline & & & & & RE Pico Caracas \\
\hline & & & & & RE El Gigante \\
\hline
\end{tabular}

El $53.93 \%$ de los registros de presencia de Magnolia en Cuba se ubican dentro del SNAP (Sistema Nacional de Áreas Protegidas). Magnolia cubensis subsp. cubensis se encontró en un mayor número de áreas protegidas (siete) entre las que se encuentran dos parques nacionales (Pico la Bayamesa, Turquino). La mayoría de los registros de M. cristalensis (98\%), M. cubensis subsp. cubensis (97.4\%), M. cubensis subsp. acunae (93.7\%), M. orbiculata $(86.79 \%)$ y M. minor (84.9\%) se encuentran dentro de algún área protegida. Magnolia oblongifolia (64.8 \%), por otra parte, posee una menor representación en áreas protegidas, mientras que Magnolia virginiana subsp. oviedoae no presenta ningún individuo dentro del Sistema Nacional de Áreas Protegidas. En los parques nacionales Alejandro de Humboldt y Pico Cristal, así como en el Área Protegida de Recursos Manejados Reserva de la Biosfera Cuchillas del Toa, conviven tres taxones de magnolias (Tabla 2).

La distribución altitudinal para Magnolia en Cuba varió desde el nivel del mar hasta los 1,648 m. Magnolia virginiana subsp. oviedoae es la que habita a una menor altitud $(6 \mathrm{~m})$ con un valor promedio de $8.54 \mathrm{~m}$, mientras que $M$. cubensis subsp. cubensis habita en altitudes de hasta 1,648 $\mathrm{m}$ (promedio de 1,397 m). Los taxones con los mayores valores promedios de altitud fueron los correspondientes a las montañas de la Sierra Maestra. Exceptuando a $M$. virginiana subsp. oviedoae cuyo rango de altura no coincide con ningún otro representante del grupo, M. minor y M. cubensis subsp. cubensis son los únicos taxones que no presentaron superposición en cuanto al rango de altitudes en la que se desarrollan. Magnolia oblongifolia presentó el mayor rango de altitudes (20-850 m) (Figura 2). El análisis de varianza no paramétrico (Kruskal-Wallis) mostró diferencias estadísticas significativas entre las altitudes promedio de los siete taxones. La prueba de Dunn permitió detectar que con excepción del par M. cubensis subsp. acunae $-M$. cristalensis $(P=0.11)$, todas las comparaciones resultaron con diferencias estadísticamente significativas $(P<0.001)$.

Todas las magnolias cubanas se encuentran amenazadas según los criterios establecidos por la UICN. Magnolia orbiculata es el único taxón que se encuentra críticamente amenazado (CR) mientras que tres taxones $(M$. cubensis subsp. acunae, M. cubensis subsp. cubensis y M. minor) se encuentran En Peligro (EN) de extinción y los otros tres (M. virginiana subsp. oviedoae, M. cristalensis y M. oblongifolia) son Vulnerables (VU) a la extinción (Tabla 3).

Los criterios en los que se sustentan dichas evaluaciones varían entre los taxones. Las categorías de M. cubensis subsp. acunae y $M$. orbiculata se basaron en la distribución geográfica representada como extensión de EP y/o AO (criterio B), mientras que $M$. cubensis subsp. cubensis y M. minor se evaluaron en función del tamaño poblacional y su tendencia (criterio C). La categorización de los tres taxones vulnerables se basó en el número conocido de individuos maduros de cada uno (criterio D). Las principales amenazas que presenta el género Magnolia en Cuba son la tala (en 5 de 7 taxones), la modificación del hábitat por la presencia de especies exóticas invasoras (4 de 7) y la existencia de plantaciones forestales (4 de 7) en varios de sus hábitats (abla 3 ). 


\section{Distribución y conservación de Magnolia en Cuba}

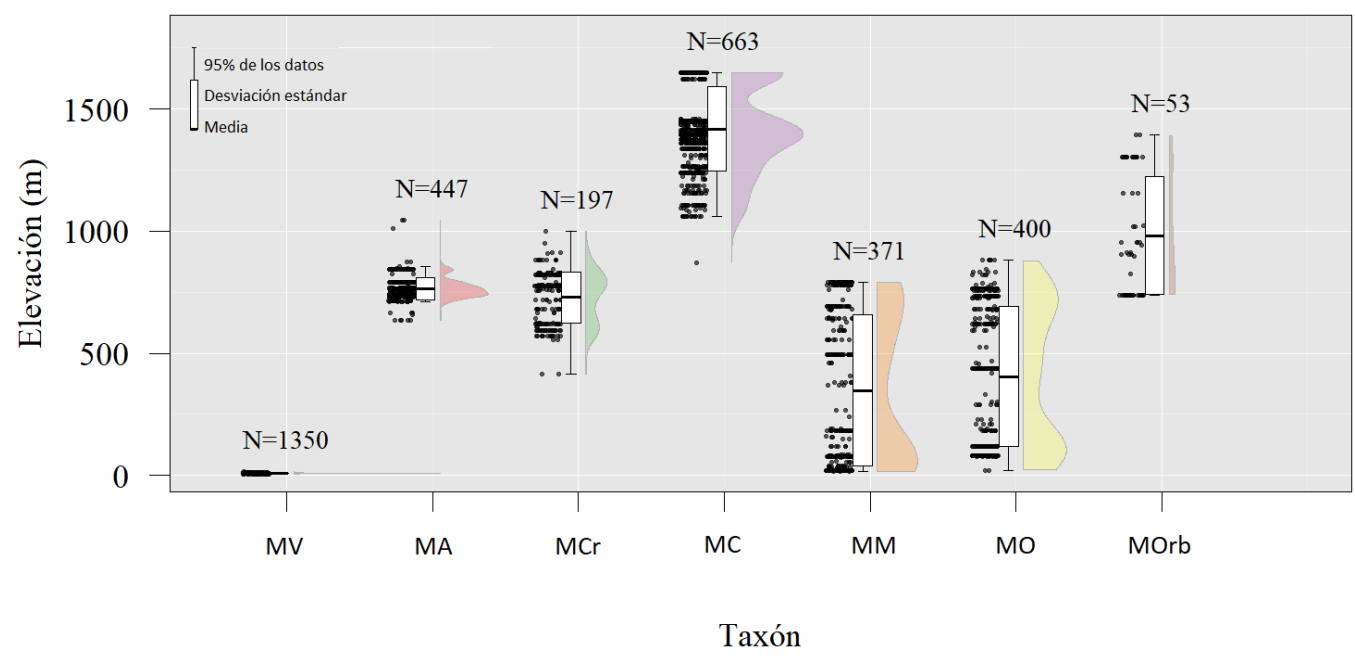

Figura 2. Comparación de los valores de altitud para los taxones cubanos de Magnolia. M. virginiana subsp. oviedoae (MV), M. cubensis subsp. acunae (MA), M. cubensis subsp. cubensis (MC), M. cristalensis (MCr), M. minor (MM), M. oblongifolia (MO) y M. orbiculata (MOrb). Número de individuos (N).

Tabla 3. Categorías, criterios y principales amenazas de los taxones cubanos de Magnolia. En Peligro Crítico de extinción (CR), En Peligro de extinción (EN), Vulnerable de extinción (VU).

\begin{tabular}{|c|c|c|c|}
\hline Taxón & Categoría & Criterio & Principales Amenazas \\
\hline $\begin{array}{l}\text { Magnolia virginiana subsp. } \\
\text { oviedoae }\end{array}$ & VU & D2 & $\begin{array}{l}\text { Actividad forestal, actividad petrolera, incremen- } \\
\text { to de los incendios, especies exóticas invasoras, } \\
\text { cambios hídricos, construcción de viales. }\end{array}$ \\
\hline $\begin{array}{l}\text { Magnolia cubensis subsp. } \\
\text { acunae }\end{array}$ & $\mathrm{EN}$ & B1ab(iii)+2ab(iii) & $\begin{array}{l}\text { Agricultura, tala, especies exóticas invasoras, } \\
\text { ganadería, áreas de recreación y turismo, áreas } \\
\text { urbanas. }\end{array}$ \\
\hline $\begin{array}{l}\text { Magnolia cubensis subsp. } \\
\text { cubensis }\end{array}$ & EN & $\mathrm{C} 2 \mathrm{a}(\mathrm{i})$ & $\begin{array}{l}\text { Tala, especies exóticas invasoras, agricultura, } \\
\text { áreas de recreación y turismo. }\end{array}$ \\
\hline Magnolia cristalensis & VU & D1 & Agricultura, minería, tala, plantaciones forestales. \\
\hline Magnolia minor & $\mathrm{EN}$ & $\mathrm{C} 2 \mathrm{a}(\mathrm{ii})$ & $\begin{array}{l}\text { Especies exóticas invasoras, minería, cambio } \\
\text { climático, plantaciones forestales industriales, } \\
\text { tala. }\end{array}$ \\
\hline Magnolia oblongifolia & VU & D1 & Minería, plantaciones forestales industriales \\
\hline Magnolia orbiculata & $\mathrm{CR}$ & B1ab(ii,iii,v);C2a(i) & $\begin{array}{l}\text { Áreas de recreación y turismo, cambio climático, } \\
\text { tala. }\end{array}$ \\
\hline
\end{tabular}




\section{Discusión}

La distribución actual de Magnolia, coincide de manera general, con las localidades descritas en la literatura para los distintos taxones (Bisse 1988, Imchanitzkaja 1991, 1993, Oviedo et al. 2006, González-Oliva et al. 2015, Palmarola et al. 2016, Testé 2018, Testé et al. 2019). El extremo más occidental de la distribución de las magnolias cubanas se encuentra representado por M. virginiana subsp. oviedoae, la cual es también la población más al sur de Magnolia virginiana (Zale 2009). Esta especie habita en terreno cenagoso y es la única magnolia nativa de Cuba que se desarrolla en ese ambiente (Testé 2018).

Hacia el centro de la isla se localiza Magnolia cubensis subsp. acunae, en un hábitat históricamente fragmentado (Hernández et al. 2020) y afectado por deforestación para ganadería y cultivo de café, tala furtiva e introducción de especies exóticas para sombra del café (González-Torres et al. 2013, Palmarola et al. 2018). Esta subespecie es el único taxón montano que no comparte hábitat con ningún otro; este podría ser el resultado de la historia evolutiva de aislamiento y de colonización del grupo en Cuba.

De hecho, el patrón de distribución de esta subespecie concuerda con lo reportado para otras especies del género en la región. Magnolia en la región del Caribe presenta de manera general una distribución geográfica discreta, pues cada especie tiene una distribución restringida a una o pocas islas o macizos montañosos (o ciénaga en el caso de M. virginiana subsp. oviedoae) dentro de una misma isla. Las especies alopátricas vecinas se encuentran aproximadamente entre 30 y $150 \mathrm{~km}$ de distancia entre sí (Veltjen 2020). Las dos excepciones a este patrón de distribución geográfica en las magnolias caribeñas se encuentran en Cuba: M. cubensis subsp. cubensis y M. orbiculata cohabitan en la Sierra Maestra, y M. cristalensis, M. minor y M. oblongifolia comparten hábitat en Nipe-Sagua-Baracoa. Sin embargo, la presencia de simpatría entre especies de la misma sección es exclusiva a M. minor y M. oblongifolia. Estas especies presentan diferente forma y tamaño de hojas, que pueden reflejar diferencias en especialización a diferentes porciones del gradiente de luminosidad en el bosque. Puede ser que la simpatría y la diferenciación de nicho este más relacionada a la edad del linaje, siendo mayor en los linajes más antiguos (basales), como lo es el caso de la sección Talauma.

Nuestros resultados confirman el marcado patrón alopátrico reportado para el género Magnolia (Vázquez-García 1994, 2015, 2016a, 2018), aunque muestra una mayor preponderancia del patrón alopátrico sobre el simpátrico (71:29 \%) con respecto de lo documentado para el Ecuador (58:42 \%) (Vázquez-García et al. 2016b, 2018). Cabe resaltar que Cuba es el único país del mundo donde se encuentran representadas las siguientes tres secciones del género Magnolia: Magnolia, Splendentes y Talauma. Lo cual se explica en parte por ubicarse en una zona de transición entre los reinos biogeográficos Holártico y Neotrópico que involucra desde clima templado cálido hasta tropicales (Morrone et al. 2017). Aunque comparte con México esta zona de transición, difiere de este último en que en Cuba si ocurre la sección Splendentes, pero no la sección Macrophylla, y viceversa.

Los valores de extensión de presencia y área de ocupación para los taxones de Magnolia se ubican de manera general dentro del rango de valores referidos en las anteriores categorizaciones (Palmarola 2015, Palmarola \& González-Torres 2015, Palmarola et al. 2015 a,b,c,d,e). Los bajos valores de área de ocupación del grupo corresponden a categorías de amenazas superiores según el criterio B de la UICN. La amplia distribución de Magnolia minor podría incidir en un menor riesgo de extinción pues tiene una mayor adaptabilidad, estos resultados se tuvieron en cuenta para el cambio en su categoría de amenaza (Hernández et al. en prensa e). Los bajos valores de EP y AO de M. virginiana subsp. oviedoae coinciden con lo reportado por Palmarola et al. (2015e) y Testé (2018). Por otra parte, los reducidos valores de EP y AO de Magnolia orbiculata difieren significativamente de lo referido por Palmarola et al. (2016). Palmarola et al. (2015d) categorizan a esta especie como Vulnerable, basado en prospecciones de campo puntuales y materiales de herbarios. Según los resultados de la presente investigación la especie es re-evaluada En Peligro Crítico de extinción.

La densidad de Magnolia virginiana subsp. oviedoae resalta sobre la del resto de las magnolias cubanas. Su alto número de individuos por $\mathrm{km}^{2}$ (450) no resulta un indicador del buen estado de la población, sino que responde a que la población se encuentra íntegramente distribuida en una baja EP $\left(3 \mathrm{~km}^{2}\right)$. El resto de las magnolias cubanas 
presentan bajos valores de densidad similar a los encontrados en la literatura para M. cubensis subsp. cubensis (Testé et al. 2019), M. dealbata Zucc. (Velazco-Macías et al. 2008) y Magnolia llanganatensis A. Vázquez \& D. A. Neill (Vázquez-García et al. 2016b).

La distribución por formaciones vegetales concuerda con la distribución preferencial de las magnolias en México (Vázquez-García 1994), Centroamérica (Vázquez-García 1994) y en general en las zonas intertropicales (Monk 1966), con sus equivalentes continentales: bosques mesófilos de montaña, bosques nubosos, bosques tropicales montanos. Esto es de esperar teniendo en cuenta la alta exigencia ambiental de las magnolias (May 1994, Fonseca-Cortés 2010). No obstante, solo dos de las magnolias cubanas (M. virginiana subsp. oviedoae y M. orbiculata) se encontraron en dos formaciones vegetales. El resto de los taxones se encontraron habitando en al menos cuatro tipos distintos de vegetación. Esto resulta lógico por el hecho de que la vegetación no es el único factor que condiciona el ambiente donde una especie se desarrolla. Muchas otras condiciones (climáticas, geográficas o topográficas) definen el hábitat característico de una especie, y consecuentemente su exigencia ambiental. Vale la pena resaltar que fueron también identificadas dos formaciones vegetales en las que Magnolia no había sido reportada para Cuba (bosque secundario y complejo de vegetación de mogote). La vegetación secundaria surge como parte de la degradación de formaciones vegetales en las que las magnolias suelen habitar (Capote \& Berazaín 1984). Por lo tanto, su presencia en este tipo de vegetación podría no garantizar su supervivencia a largo plazo, ya que este grupo tiene una baja regeneración natural que incluso puede llegar a ser menor en áreas con estas características. Sin embargo, las magnolias son elementos estructurales de los bosques donde habitan (Wiehle et al. 2016) y sirven como hábitat a otras especies (Shen et al. 2020, Promputtha et al. 2017). Por lo tanto, tiene sentido utilizarlas como punto de partida para la recuperación de los bosques primarios, en zonas donde estos han sido degradados a bosques secundarios. Para lograrlo, deberían enfocarse las acciones de reforzamiento poblacional que forman parte de los planes de recuperación de estos taxones en este tipo de zonas. Otras áreas donde pueden enfocarse estos esfuerzos son los ecosistemas agroforestales. Por ejemplo, el reforzamiento con individuos de Magnolia cubensis subsp. acunae en los cafetales de Topes de Collantes, permitirá la sustitución de especies exóticas actualmente utilizadas como sombra del café por esta subespecie nativa, según lo propuesto por González-Torres et al. (2013).

El hecho de que el bosque siempreverde mesófilo sea la formación vegetal más común concuerda con lo planteado por Bisse (1998), Palmarola et al. (2016) y González-Torres et al. (2016). La preferencia de las magnolias por esta formación vegetal podría tener repercusiones negativas en sus poblaciones. Por una parte, debido a que este tipo de bosque es considerado altamente amenazado por deforestación, fragmentación, degradación (Cabrera \& Ramírez 2007, Cuesta et al. 2009, Tejedor-Garavito et al. 2012) e impactos potenciales del cambio climático (Williams-Linera 2007, Fuentes et al. 2019). Las especies que habitan en este tipo de bosques tienen un alto grado de especialización, por lo que su adaptación ante la pérdida en área y calidad de sus hábitats puede ser difícil de alcanzar. Estudios como el de Monterroso-Rivas et al. (2013) evaluaron la respuesta de varias especies mexicanas ante diferentes escenarios de cambio climático, concluyendo que muchas verán reducidas sus superficies óptimas. En el caso de las magnolias, Vásquez-Morales (2015) plantea que al cambiar el clima de los bosques siempreverdes estas especies tendrían que desplazarse a zonas de mayor altitud.

La amenaza real que representa la pérdida de hábitat en estas especies, se puede ver reducida en cierta medida por la inclusión de más del $50 \%$ de los individuos conocidos de las magnolias cubanas en áreas protegidas. Este hecho favorece su conservación a largo plazo, ya que según Kremen et al. (2008), las áreas protegidas tienen gran utilidad en el establecimiento de prioridades de conservación y permiten optimizar los fondos. Magnolia virginiana subsp. oviedoae no posee ningún individuo en áreas protegidas, lo que debería tenerse en cuenta en el diseño de las estrategias para su conservación. Aunque al establecer prioridades para el reforzamiento poblacional de los siete taxones se deben tener en cuenta las zonas de presencia que se correspondan con áreas protegidas, la conservación no debe restringirse solo a las actuales áreas (Granado-Pérez 2015). Los esfuerzos deben ir más allá de las barreras legales del SNAP y enfocarse en las áreas con mayor idoneidad y hábitats adecuados (Rodríguez-Santana 2010).

La distribución altitudinal de Magnolia en Cuba coincide con lo reportado por Bisse (1988), Palmarola et al. (2016), Palmarola et al. (2018), Testé (2018). La altitud máxima alcanzada por Magnolia cubensis subsp. cubensis es 
ligeramente inferior a lo descrito por Palmarola et al. (2015b), quien exponen que esta puede habitar hasta los 1,800 $\mathrm{m}$. Por otra parte, la altitud promedio de Magnolia minor, se encuentra muy por debajo de lo anteriormente descrito, ya que Bisse (1988) y Palmarola et al. (2016) plantean que las magnolias en Cuba se encuentran por encima de los $700 \mathrm{~m}$. Las diferencias estadísticas significativas encontradas entre las altitudes promedio pueden estar dadas por: 1) los taxones habitan en varias formaciones vegetales, que por lo general se encuentran a distintas altitudes, 2) una misma formación vegetal puede encontrase a distintas altitudes en diferentes macizos (Capote \& Berazaín 1984).

En sentido general, la distribución altitudinal responde a lo descrito para el género en el Caribe. Al igual que lo encontrado en Cuba para M. cubensis subsp. acunae, M. cubensis subsp. cubensis y M. cristalensis, el resto de los taxones de la Subsección Splendentes en el Caribe, se distribuyen a grandes altitudes en los macizos montañosos Massif de La Hotte, Massif du Nord y Massif de Cahos (en Haití), en la Cordillera Central y la Sierra Bahoruco (en República Dominicana), así como en la Cordillera Central y la Sierra de Luquillo (en Puerto Rico). Por otra parte, el único taxón caribeño de la Subsección Talauma que se distribuye fuera de Cuba (M. dodecapetala), habita en altitudes menores en las islas San Vicente, Santa Lucía, Martinica, Dominica y Guadalupe de las Antillas Menores, tal y como se observa en M. minor y M. oblongifolia (Veltjen 2020). Los valores encontrados para las magnolias cubanas se encuentran por debajo de los valores de altitud reportados para otras especies de Magnolia en el Neotrópico (Vásquez-Morales et al. 2014, Shalisko et al. 2018, Vásquez-García et al. 2018), lo cual puede estar causado en parte por las mayores altitudes de la plataforma continental. Este comportamiento puede ser también un reflejo de diferencias filogenéticas entre los taxones, que pueden relacionarse con diferencias adaptativas. De manera general, los representantes de la Sección Talauma tienden a adaptarse a climas más cálidos y por lo tanto se distribuyen a altitudes menores que los representantes de la Sección Splendentes. Con excepción de Magnolia minor las categorías de amenaza del resto de las magnolias cubanas cambiaron con respecto a la categorización anterior (Palmarola 2015, Palmarola \& González-Torres 2015, Palmarola et al. 2015a, b,c,d,e). Esto se debe fundamentalmente a que las evaluaciones realizadas en 2015 se basaban en inferencias en cuanto al tamaño poblacional de los distintos taxones, con poco conocimiento de sus poblaciones y no se utilizaban herramientas como GeoCAT. Desde esa fecha al presente se han identificado nuevos sitios de presencia (e.g., Tres Palmas para Magnolia acunae subsp. acunae; Monte Fresco para M. cristalensis; La Melba para M. minor) y se ha profundizado en el conocimiento de las poblaciones y amenazas de estos taxones mediante estudios poblacionales (Palmarola et al. 2017, Testé 2018, Testé et al. 2019, MartínezÁlvarez et al. 2020), que han permitido una evaluación más profunda de su estado de conservación.

\section{Agradecimientos}

Este trabajo fue realizado gracias al apoyo de Planta! (www.planta.ngo), la Sociedad Cubana de Botánica, el Jardín Botánico Nacional y la Facultad de Biología (Universidad de La Habana), la Empresa Nacional para la Protección de la Flora y la Fauna, el Centro Nacional de Áreas Protegidas, Fauna and Flora International, Arboretum Wespelaar, Fondation Franklinia, Whitley Fund for Nature y Magnolia Society International. Finalmente estamos agradecidos a todas las personas que permitieron el trabajo de campo, especialmente a Arlet Rodríguez-Meno, Eldis R. Bécquer, Jose Luis Gómez, José Luis Moscoso, Leandro Galano, Norlys Albelo, Oliver Valle y Sergio Hernández.

\section{Literatura citada}

Bachman S, Moat J, Hill AW, de la Torre J, Scott B. 2011. Supporting Red List threat assessments with GeoCAT: geospatial conservation assessment tool. In: Smith V, Penev L, eds. e-Infrastructures for Data Publishing in Biodiversity Science. ZooKeys 150: 117-126. ISBN: 9789546426192; DOI: https://doi.org/10.3897/zookeys.150.2109

Bisse J. 1988. Árboles de Cuba. Ciudad de La Habana: Editorial Científico-Técnica.

Cabrera E, Ramírez D. 2007. Estado actual y cambio en los ecosistemas de los Andes colombianos: 1985-2005. In: Armenteras-Pascual D, Rodríguez-Eraso N. eds. Monitoreo de los Ecosistemas Andinos 1985-2005: Síntesis y Perspectivas. Bogotá, D.C. Colombia: Instituto de Investigación Alexander von Humbolt, pp 41-63. ISBN: 978-9-58834-312-9 
Capote R, Berazaín R. 1984. Clasificación de las formaciones vegetales de Cuba. Revista del Jardín Botánico Nacional. La Habana 5: 27-75.

Cires E, De Smet Y, Cuesta C, Goetghebeur P, Sharrock S, Gibbs D, Olfield, S, Kramer, A, Samain MS. 2013. Gap analyses to support ex situ conservation of genetic diversity in Magnolia, a flagship group. Biodiversity and Conservation 22: 567-590. DOI: https://doi.org/10.1007/s10531-013-0450-3

CNAP [Centro Nacional de Áreas Protegidas]. 2014. Plan del Sistema Nacional de Áreas Protegidas 2014-2020. Centro Nacional de Áreas Protegidas. La Habana, Cuba, 200 pp. ISBN: 978-959-287-049-9

Cuesta F, Peralvo M, Valarezo N. 2009. Los bosques montanos de los Andes tropicales. Una evaluación regional de su estado de conservación y de su vulnerabilidad a efectos del cambio climático. Quito, Ecuador: Programa Regional ECOBONA. ISBN: 978-9942-9966-0-2

Esri [Environmental Systems Research Institute]. 2013. ArcGIS 10.0. Redlands, California

Estrada R, Martín G, Martínez P, Rodríguez S, Capote R, Reyes I, Galano S, Cabrera C, Martínez C, Mateo L, Guerra Y, Batte A, Coya L. 2019. Mapa (BD-SIG) de vegetación natural y seminatural de Cuba. Atlas Nacional de Cuba LX Aniversario. Instituto de Geografía Tropical. https://atlas.gocuba.cu/flora/ (accessed February 1, 2021).

Fonseca-Cortés A. 2010. Estructura poblacional y distribución espacial de Magnolia colombiana (Lozano) Govaerts en el Parque Nacional Natural Cueva de los Guacharos y su zona de influencia. Huila, Colombia. BSc Thesis. Universidad de La Amazonia.

Fuentes IM, González-Oliva L, Baró I, González-Echeverría MT, Mancina CA. 2019. Efecto potencial del cambio climático sobre la distribución de plantas asociadas a bosques húmedos del oriente de Cuba. Acta Botánica Cubana 218: 160-170.

González-Oliva L, González-Torres LR, Palmarola A, Barrios D, Testé E. eds. 2015. Categorización de taxones de la flora de Cuba. Bissea 9: 3-307.

González-Torres, LR, Palmarola A, Bécquer ER, BerazaínR, Barrios D, Gómez JL. 2013. Top 50: Las 50 plantas más amenazadas de Cuba. Bissea 7: 1-107.

González-Torres LR, Palmarola A, González Oliva L, Bécquer ER, Testé E, Barrios D. eds. 2016. Lista roja de la flora de Cuba. Bissea 10: 1-352.

Granado-Pérez L. 2015. Estructura poblacional, distribución geográfica y conservación de Magnolia cubensis subsp. acunae (Magnoliaceae). BSc Thesis. Universidad de La Habana.

Greuter W, Rankin R. 2017. Plantas Vasculares de Cuba Inventario preliminar. Segunda edición, actualizada, de Espermatófitos de Cuba con inclusión de los Pteridófitos. Berlín \& La Habana: Botanischer Garten \& Botanisches Museum Berlin-Dahlem \& Jardín Botánico Nacional, Universidad de La Habana. DOI: http://dx.doi.org/10.3372/ cubalist.2017.1

Gutiérrez-Lozano M, Sánchez-González A, Vázquez-García JA, López-Mata L, Octavio-Aguilar P. 2020. Diferenciación morfológica poblacional de Magnolia rzedowskiana (Magnoliaceae): especie endémica en peligro de extinción de la sierra Madre Oriental, México. Revista Mexicana de Biodiversidad 91: e913101. DOI: http://dx.doi. org/10.22201/ib.20078706e.2020.91.3101

Hernández M, Palmarola A, Veltjen E, Asselman P, Testé E, Larrion I, González-Torres LR. 2020. Population structure and genetic diversity of Magnolia cubensis subsp. acunae (Magnoliaceae): effects of habitat fragmentation and implication for conservation. Oryx 54: 1-9. DOI: https://doi.org/10.1017/s003060531900053x

Hernández M, Testé E, Palmarola A. Magnolia virginiana subsp. oviedoae - Hoja de taxón. Bissea 14. en prensa a.

Hernández M, Testé E, Albelo N, Valle O, Simón R, Palmarola A. Magnolia cubensis subsp. acunae - Hoja de taxón. Bissea 14: en prensa b.

Hernández M, Testé E, Albelo N, Valle O, Simón R, Palmarola A. Magnolia cubensis subsp. cubensis - Hoja de taxón. Bissea 14: en prensa c.

Hernández M, Testé E, Gómez-Hechavarria JL, Palmarola A, González-Torres LR, Simón R. Magnolia cristalensis - Hoja de taxón. Bissea 14: en prensa d. 
Hernández M, Testé E, Palmarola A, Simón R, González-Torres LR. Magnolia minor - Hoja de taxón. Bissea 14: en prensa e.

Hernández M, Testé E, Simón R, Palmarola A, González-Torres LR. Magnolia oblongifolia - Hoja de taxón. Bissea 14: en prensa $\mathrm{f}$.

Hernández M, Testé E, Simón R, Palmarola A, González-Torres LR. Magnolia orbiculata - Hoja de taxón. Bissea 14: en prensa $g$

Imchanitzkaja NN. 1991. Rod Magnolia L. (Magnoliaceae) vo flore Kuby. Novosti Sistematiki Visshikh Rasteniy 28 : 58-77.

Imchanitzkaja NN. 1993. Genus Talauma A. L. Juss. (Magnoliaceae) in flora Cubae. Novosti Sistematiki Visshikh Rasteniy 29: 76-84.

IUCN [Standards and Petitions Committee]. 2019. Guidelines for Using the IUCN Red List Categories and Criteria. Version 14. Standards and Petitions Committee. http://www.iucnredlist.org/documents/RedListGuidelines. pdf (accessed February 1, 2021).

Kremen C, Cameron A, Moilanen A, Phillips SJ, Thomas CD, Beentje H, Dransfield J, Fisher BL, Glaw F, Good TC, Harper GJ, Hijmans RJ, Lees DC, Louis EJ, Nussbaum RA, Raxworthy CJ, Razafimpahanana A, Schatz GE, Vences M, Vieites DR, Wright PC, Zjhra ML. 2008. Aligning conservation priorities across taxa in Madagascar with high-resolution planning tools. Science 320: 222-225. DOI: https://doi.org/10.1126/science.1155193

Li J, Wu Y, Wu T, Cao L, Chen J, Qiu Z, Liu P. 2017. Significance of stump-sprouting for the population size structure and spatial distribution patterns of endangered species, Magnolia cylindrical. Polish Journal Ecology 65: 247- 257.

Martínez-Álvarez G, Hernández M, Quintana J, González-Torres LR. 2020. Estructura etaria de Magnolia cristalensis en El Toldo, Parque Nacional Alejandro de Humboldt. Revista del Jardín Botánico Nacional 41: 87-89.

May T. 1994. Regeneración de la vegetación arbórea y arbustiva en un terreno de cultivos abandonado durante 12 años en la zona de bosques húmedos montanos (Reserva Científica Ébano Verde, Cordillera Central, República Dominicana). Moscosoa 8: 131-149.

Menon S, Choudhury BI, Khan ML, Peterson AT. 2010. Ecological niche modeling and local knowledge predict new populations of Gymnocladus assamicus a critically endangered tree species. Endangered Species Research 11: 175-181. DOI: https://doi.org/H10.3354/esr00275

Monk CD. 1966. An Ecological Study of Hardwood Swamps in North-Central Florida. Ecology 46: 649-654. DOI: https://doi.org/10.2307/1933947

Monterroso-Rivas AI, Gómez-Díaz JD, Tinoco-Rueda JA. 2013. Bosque mesófilo de montaña y escenarios de cambio climático: una evaluación en Hidalgo, México. Revista Chapingo. Serie Ciencias Forestales y del Ambiente 19: 29-43. DOI: https://doi.org/10.5154/r.rchscfa.2012.03.029

Morrone JJ, Escalante T, Rodríguez-Tapia G. 2017. Mexican biogeographic provinces: Map and shaperfiles. Zootaxa 4277: 277-279. DOI: https://doi.org/10.11646/zootaxa.4277.2.8

Muñiz-Castro MA, Castro-Félix P, Carranza-Aranda AS, Vázquez-García JA, Santerre A. 2020. Population genetics, species boundaries, and conservation in the Magnolia pacifica species complex along a continentality and moisture gradient in western Mexico. Botanical Sciences 98: 500-516. DOI: https://doi.org/10.17129/botsci.2551

Oviedo R, Palmarola A, Gómez N, González-Torres LR. 2006. Primer reporte de Magnolia virginiana (Magnoliaceae) en Cuba. Revista del Jardín Botánico Nacional 27: 137-139.

Palmarola A. 2015. Magnolia minor. Bissea 9: 410.

Palmarola A, González Torres LR. 2015. Magnolia oblongifolia. Bissea 9: 405.

Palmarola A, González Torres LR, Granado L, Hernández M. 2015a. Magnolia cubensis subsp. acunae. Bissea 9: 405.

Palmarola A, González Torres LR, Hernández M, Molina Y, Gordillo M, Granado L, Cruz DD. 2015b. Magnolia cubensis subsp. cubensis. Bissea 9: 406-407.

Palmarola A, González Torres LR, Gómez-Hechavrría JL, Hernández M. 2015c. Magnolia cristalensis. Bissea 9 : 403-404. 
Palmarola A, González Torres LR, Hernández M. 2015d. Magnolia orbiculata. Bissea 9: 412.

Palmarola A, González Torres LR, Hernández M. 2015e. Magnolia virginiana subsp. oviedoae. Bissea 9: 408-409.

Palmarola A, Romanov MS, Bobrov A, González-Torres LR. 2016. Las magnolias de Cuba: Talauma - problemática taxonómica y nomenclatural. Revista del Jardín Botánico Nacional. 37: 1-10.

Palmarola A, Testé E, Gómez-Hechavarría JL, González-Torres LR. 2017. Estructura etaria de dos magnolias cubanas en Alto de Mina Iberia: Magnolia oblongifolia y M. cristalensis. Revista del Jardín Botánico Nacional. 38: $39-142$.

Palmarola A, Granado L, Testé E, Hernández M, Albelo N, González-Torres LR. 2018. Estructura poblacional y distribución de Magnolia cubensis subsp. acunae (Magnoliaceae). Revista del Jardín Botánico Nacional. 39: 103-111.

Paredes-García DM, Ramirez-Batista A, Martínez-Morales MA. 2011. Distribución y representatividad de las especies del género Crotalus en las áreas naturales protegidas de México. Revista Mexicana de Biodiversidad 82: 689-700. DOI: http://dx.doi.org/10.22201/ib.20078706e.2011.2.464

Promputtha I, McKenzie EH, Tennakoon DS, Lumyong S, Hyde KD. 2017. Succession and natural occurrence of saprobic fungi on leaves of Magnolia liiifera in a tropical forest. Cryptogamie, Mycologie 38: 213-225. DOI: https://doi.org/10.7872/crym/v38.iss2.2017.213

R Core Team 2020. R: A language end environment for statistical computing. R Foundation for Statistical Computing. Vienna, Austria. http://www.R-project.org/

Rivers M, Beech E, Murphy L, Oldfield S. 2016. The Red List of Magnoliaceae - revised and extended. Richmond, UK: Botanic Gardens Conservation International. ISBN: 978-1-905164-64-6.

Rodríguez-Santana F. 2010. Distribución, migración y conservación de las aves rapaces del orden Falconiformes en Cuba. PhD Thesis. Universidad de Alicante.

Sánchez-Velásquez LR, Pineda-López MR, Vásquez-Morales SG, Avendaño-Yáñez ML. 2016. Ecology and conservation of endangered species: The case of magnolias. In: Quinn M, ed. Endangered Species. USA: Nova Sciences Publishers, Inc. 63-84. ISBN: 978-1-63484-404-8.

Shalisko V, Vázquez-García JA, Villalobos-Arámbula AR, Muñiz-Castro MA. 2018. Vulnerability to climate change for narrowly ranged species: the case of Ecuadorian endemic Magnolia mercedesiarum. BioRxiv 482000. https:// doi.org/10.1101/482000

Shen Q, Yang J, Su Z, Xiao W, Wang Y, Cui X. 2020. Comparative analysis of fungal diversity in rhizospheric soil from wild and reintroduced Magnolia sinica. Plants 9: 600. DOI: https://doi.org/10.3390/plants9050600

Tejedor-Garavito N, Álvarez E, Arango-Caro S, Araujo-Murakami A, Blundo C, Boza-Espinoza TE, La Torre Cuadros MA, Gaviria J, Gutierez N, Jorgensen, PM, León, B, López Camacho R, Malizia L, Millán B, Moraes M, Pacheco S, Rey-Benayas JM, Reynel C, Timaná de la Flor M, Ulloa-Ulloa C, Vacas-Cruz O, Newton AC. 2012. Evaluación del estado de conservación de los bosques montanos en los Andes Tropicales. Ecosistemas 21: 148166.

Testé E. 2018. Variación en la estructura y la ecología espacial de la población de Magnolia virginiana subsp. oviedoae (Magnoliaceae), Matanzas, Cuba. MSc. Thesis. Universidad de La Habana.

Testé E, Gordillo M, Palmarola A, Hernández M, González-Torres LR. 2019. Estructura poblacional de Magnolia cubensis subsp. cubensis (Magnoliaceae) en el Paisaje Natural Protegido Gran Piedra. Revista del Jardín Botánico Nacional. 40: 19-21

Thiers B. 2020. [continuously updated]. Index Herbariorum: A global directory of public herbaria and associated staff. US, New York: New York Botanical Garden's Virtual Herbarium. http://www.sweetgum.nybg.org/science/ $\underline{\mathrm{ih} /}$ (accessed February 1, 2021).

Vásquez-Morales SG. 2015. Demografía, cambio climático y bioprospección: una aproximación Integral para la conservación de Magnolia schiedeana Schltl. PhD Thesis. Universidad Veracruzana.

Vásquez-Morales SG, Téllez-Valdés O, Pineda-López MR, Sánchez-Velásquez LR, Flores-Estevez N, Viveros-Viveros H. 2014. Effects of climate change on the distribution of Magnolia schiedeana: a threatened species. Botanical Sciences 92: 575-585. DOI: https://doi.org/10.17129/botsci.116 
Vázquez-García JA. 1994. Magnolia (Magnoliaceae) in México and Central America: a Synopsis. Brittonia 46: 1-23. DOI: http://dx.doi.org/10.2307/2807454

Vázquez-García JA, Domínguez-Yescas R, Pedraza-Ruiz R, Sánchez-González A, Muñiz-Castro MA. 2015. Magnolia rzedowskiana (Magnoliaceae), a new species of section Macrophylla from the central Sierra Madre Oriental, México. Acta Botanica Mexicana 112: 19-36. DOI: http://dx.doi.org/10.21829/abm112.2015.1086

Vázquez-García JA, Neill DA, Asanza M, Pérez AJ, Arroyo F, Dahua-Machoa A, Merino-Santi RE. 2016a. Magnolias de Ecuador: en riesgo de extinción. Ecuador, Puyo: Universidad Estatal Amazónica, Universidad de Guadalajara-CUCBA, Pontificia Universidad Católica de Ecuador, Universidad Nacional Agraria La Molina. ISBN: 978-9942-932-18-1.

Vázquez-García JA, Neill DA, Recalde F, Asanza M. 2016b. Magnolia llanganatensis (Subsect. Talauma, Magnoliaceae), una especie nueva de Tungurahua y clave para las especies Magnolia de Ecuador. Botanical Sciences 94: 593-602. DOI: https://doi.org/10.17129/botsci.435

Vázquez-García A, Neill DA, Shalisko V, Arroyo F, Merino-Santi RE. 2018. Magnolia mercedesiarum (subsect. Talauma, Magnoliaceae): a new Andean species from northern Ecuador, with insights into its potential distribution. Phytotaxa 348: 254-268. https://doi.org/10.11646/phytotaxa.348.4.2

Velazco-Macías CG, Foroughbakhch-Pournavab R, Alanís-Flores GJ, Alvarado-Vázquez MA. 2008. Magnolia dealbata en Nuevo León, México. Revista Mexicana de Biodiversidad 79: 459- 463. DOI: http://dx.doi.org/10.22201/ ib.20078706e.2008.002.554

Veltjen E. 2020. The Caribbean Magnolia species (Magnoliaceae): Assessment of the genetic diversity and the underlying evolutionary history. PhD Thesis. Universidad de Gante.

Wang YB, Liu BB, Nie ZL, Chen HF, Chen FJ, Fligar RB, Wen J. 2020. Major clades and a revised classification of Magnolia and Magnoliaceae based on whole plastid genoma sequences via genoma skimming. Journal of Systematic and Evolution 58: 673-695. DOI: https://doi.org/10.1111/jse.12588

Wiehle M, Vornam B, Wesche K, Goenster S, Buerkert A. 2016. Population structure and genetic diversity of Populus laurifolia in fragmented riparian gallery forest of the Mongolian Altai Mountains. Flora 224: 112-122. DOI: http://dx.doi.org/10.1016/j.flora.2016.07.004

Williams-Linera G. 2007. El bosque de niebla del centro de Veracruz. Ecología, historia y destino en tiempos de fragmentación y cambio climático. México: Instituto Nacional de Ecología (INECOL). Comisión Nacional para el Conocimiento y Uso de la Biodiversidad (CONABIO). ISBN: 9707091010

Zale P. 2009. Studies on the optimization of breeding potential and development in Magnolia virginiana L. MSc. Thesis. Universidad Estatal de Ohio.

Editor de sección: Eduardo Ruiz Sánchez

Contribución de los autores:

AP, RS y ET concibieron la idea original, diseñaron la investigación, georreferenciaron los registros de presencia y analizaron los datos. MH georreferenció registros de presencia y analizó los datos. AS y YM, georreferenciaron gran parte de los registros de presencia. LRGT concibió la idea original y diseño la investigación. Todos los autores contribuyeron de manera activa en la redacción, discusión de los resultados y revisión del manuscrito. 